\title{
Laser-Induced Photoreduction for Selective Tuning of Oxidation State and Crystal Structure of Hematite Nanorods
}

Heejung Kong ${ }^{1}$, Suwon Hwang ${ }^{1}$, Junhee Lee ${ }^{1}$, Suk Woo Park ${ }^{2}$, Yong-Su Han ${ }^{2}$, and Junyeob

$$
Y e O^{1,3, *}
$$

${ }^{1}$ Novel Applied Nano Optics Lab., Department of Physics, Kyungpook National University, 80, Daehak-ro, Buk-gu, Daegu 41566, Republic of Korea

${ }^{2}$ KNU Instrumental Analysis Center, Kyungpook National University, 80, Daehak-ro, Bukgu, Daegu 41566, Republic of Korea

${ }^{3}$ Department of Hydrogen and Renewable Energy, Kyungpook National University, 80, Daehak-ro, Buk-gu, Daegu 41566, Republic of Korea

* Corresponding author: junyeob@knu.ac.kr 


\section{Band gap estimation using a Tauc plot}

A Tauc plot is normally used to estimate the bandgap of a semiconductor thin film. First, the absorbance $(A)$ is calculated from the measured transmittance $(T)$ using the Beer-Lambert law:

$$
A=-\log _{10} T
$$

Next, the attenuation coefficient (or absorption coefficient, $\alpha$ ) with respect to wavelength is calculated from $A$ as follows:

$$
\alpha=(2.303 \times A) / t
$$

where $t$ denotes the thickness of the sample. The wavelength $(\lambda)$ can be converted to photon energy $(E)$ with the unit of eV as follows:

$$
\begin{aligned}
& v=c / \lambda \\
& E=h v
\end{aligned}
$$

where $v$ denotes the frequency of photon, $c$ denotes the speed of light $\left(3 \times 10^{8} \mathrm{~m} \mathrm{~s}^{-1}\right)$, and $h$ denotes Planck's constant $\left(6.626 \times 10^{-34} \mathrm{~J} \mathrm{~s}^{-1}\right)$. A Tauc plot can be plotted by setting its $\mathrm{x}$-axis to be $\mathrm{E}$ and $\mathrm{y}$-axis to be $(\alpha h v)^{n}$, where $n$ is 2 for a semiconductor with the direct bandgap and $1 / 2$ for a semiconductor with an indirect bandgap such as hematite. When the tangents of the curve are drawn on both sides of the curve with different slopes, as shown in Figure 2d, the xcoordinate at which the two tangents meet is the estimated bandgap. 


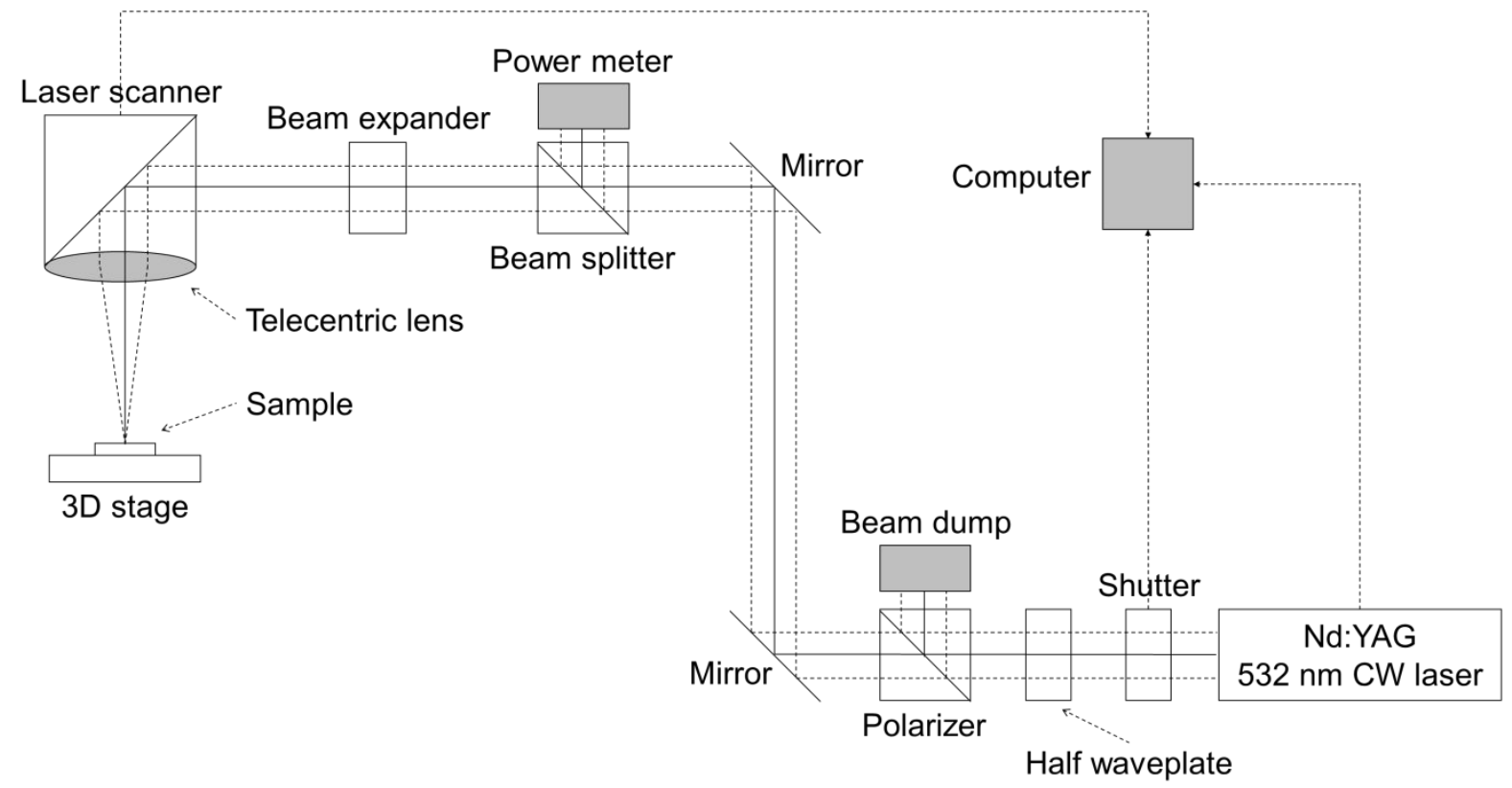

Figure S1. The optical system used in this study. 


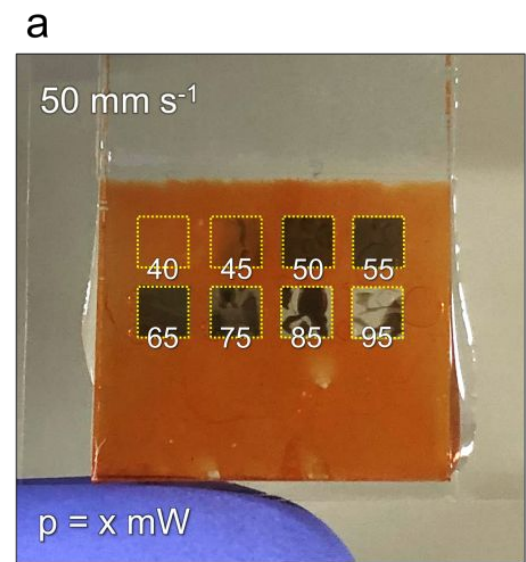

b

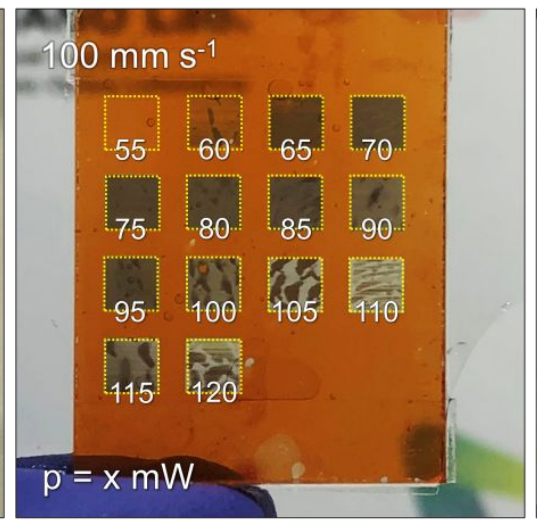

C

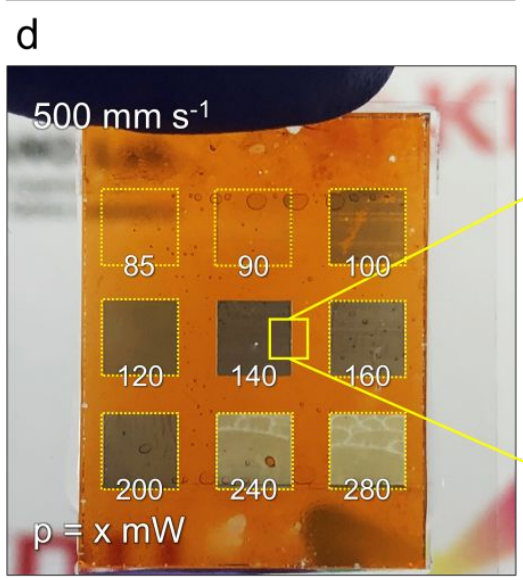

e

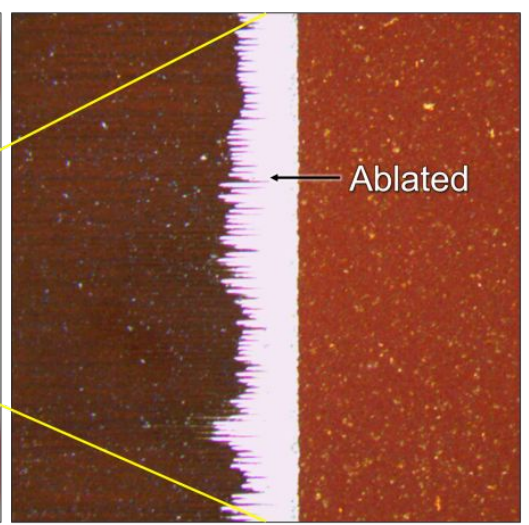

f
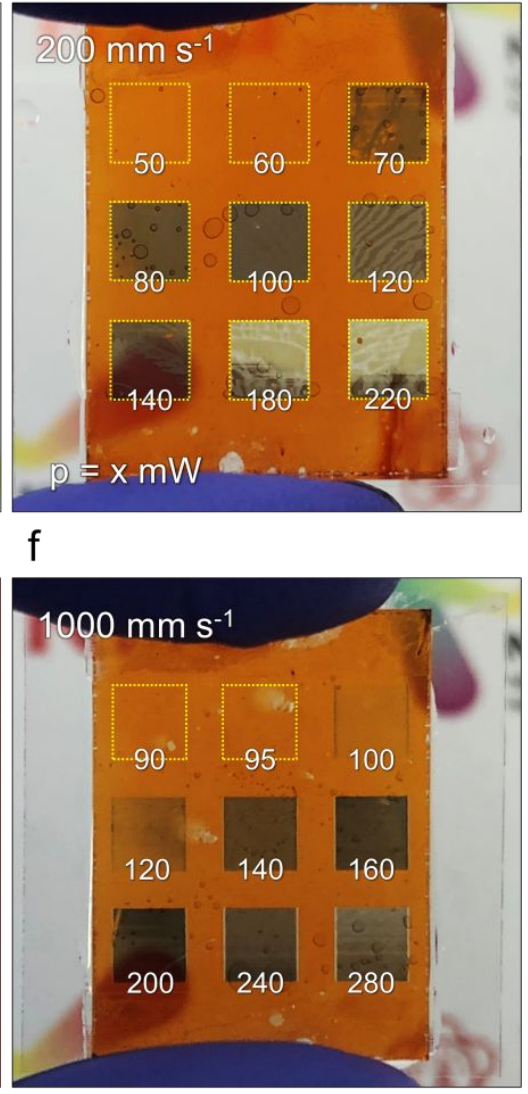

g

$\mathrm{h}$
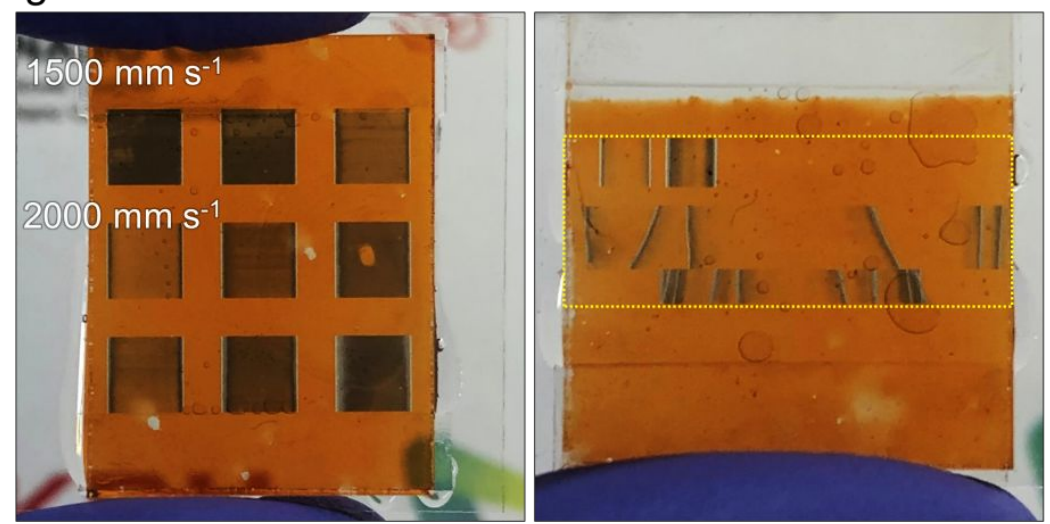

Figure S2. The influence of various scanning speeds on the LIPR process of the hematite NRs.

The pictures of the hematite samples that had undergone the LIPR processes with the scanning speeds of (a) $50 \mathrm{~mm} \mathrm{~s}^{-1}$, (b) $100 \mathrm{~mm} \mathrm{~s}^{-1}$, (c) $200 \mathrm{~mm} \mathrm{~s}^{-1}$, (d) $500 \mathrm{~mm} \mathrm{~s}^{-1}$, (f) $1000 \mathrm{~mm} \mathrm{~s}^{-1}$, (g) 1500 or $2000 \mathrm{~mm} \mathrm{~s}^{-1}$, and (h) $20000 \mathrm{~mm} \mathrm{~s}^{-1}$. (e) The optical microscope image of the area marked by the yellow triangle in (d), showing the ablation of the edge. 


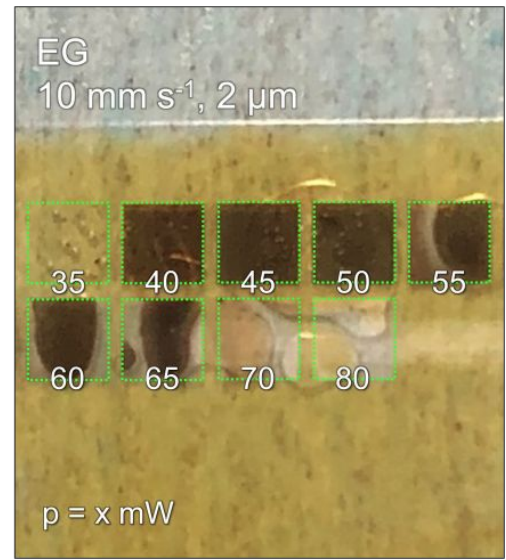

Figure S3. The picture of the akaganeite NRs sample that had undergone the LIPR process with various laser powers. The approximate laser-scanned areas are marked by the green dotted lines and each number refers to the laser powers used for the scanning the area. 


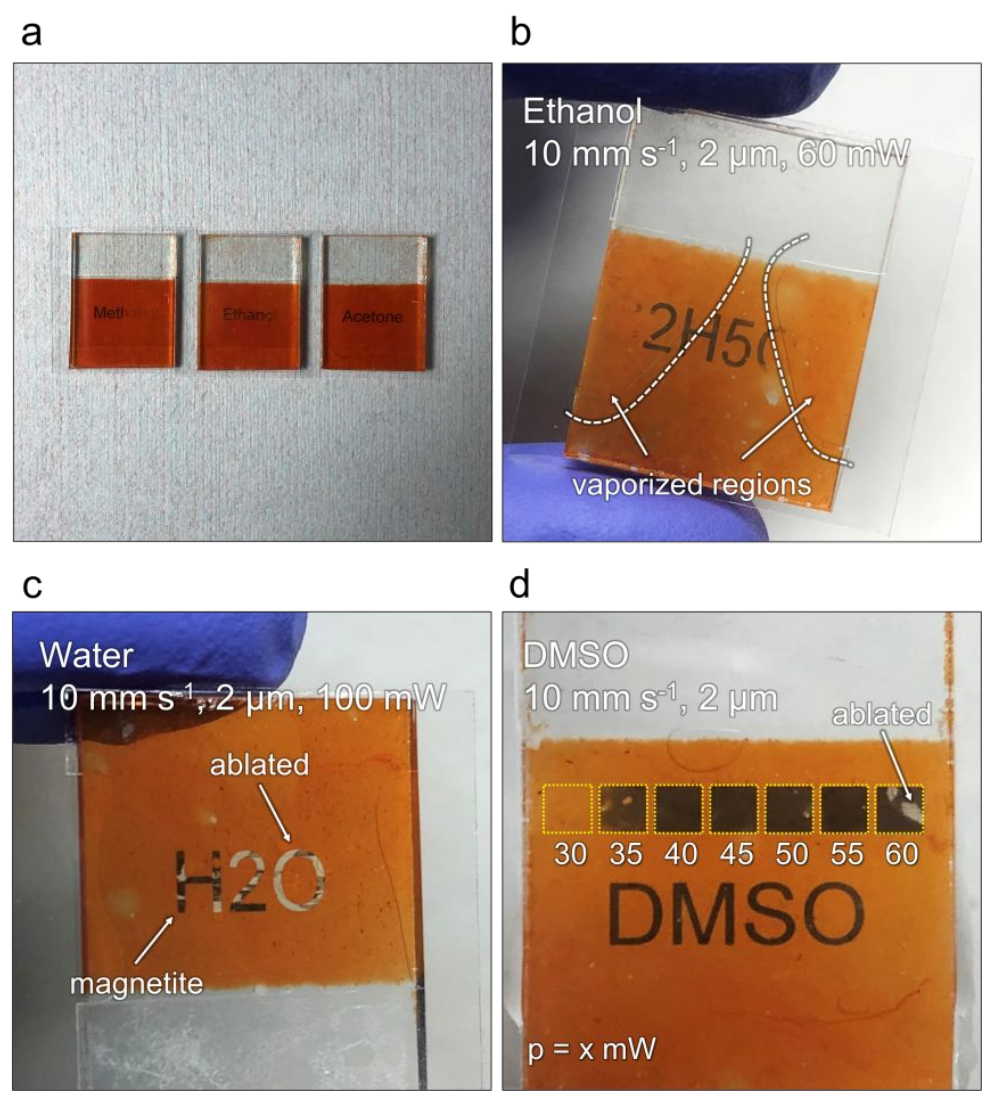

Figure S4. (a) The picture of the hematite samples after the LIPR processes with different reducing agents. From the left, methanol, ethanol, and acetone were employed as reducing agents, respectively. (b)-(d) The pictures of the hematite NRs samples that had undergone the LIPR processes with various reducing agents of (b) ethanol, (c) DI water, and (d) dimethyl sulfoxide (DMSO). 


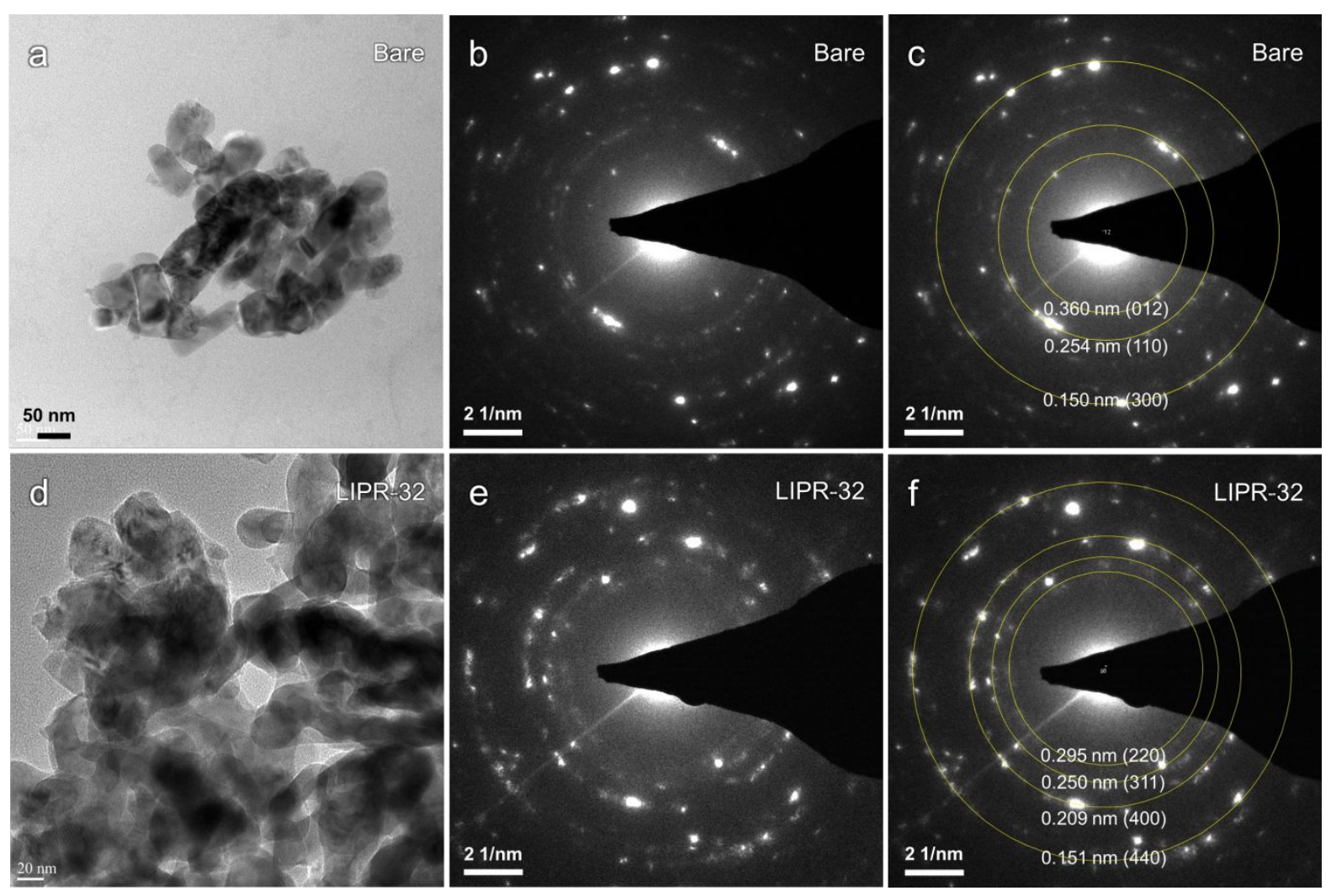

Figure S5. The low magnification TEM images of (a) the bare sample and (d) the LIPR-32.

The SAED patterns of (b)-(c) the bare sample and (e)-(f) the LIPR-32. 

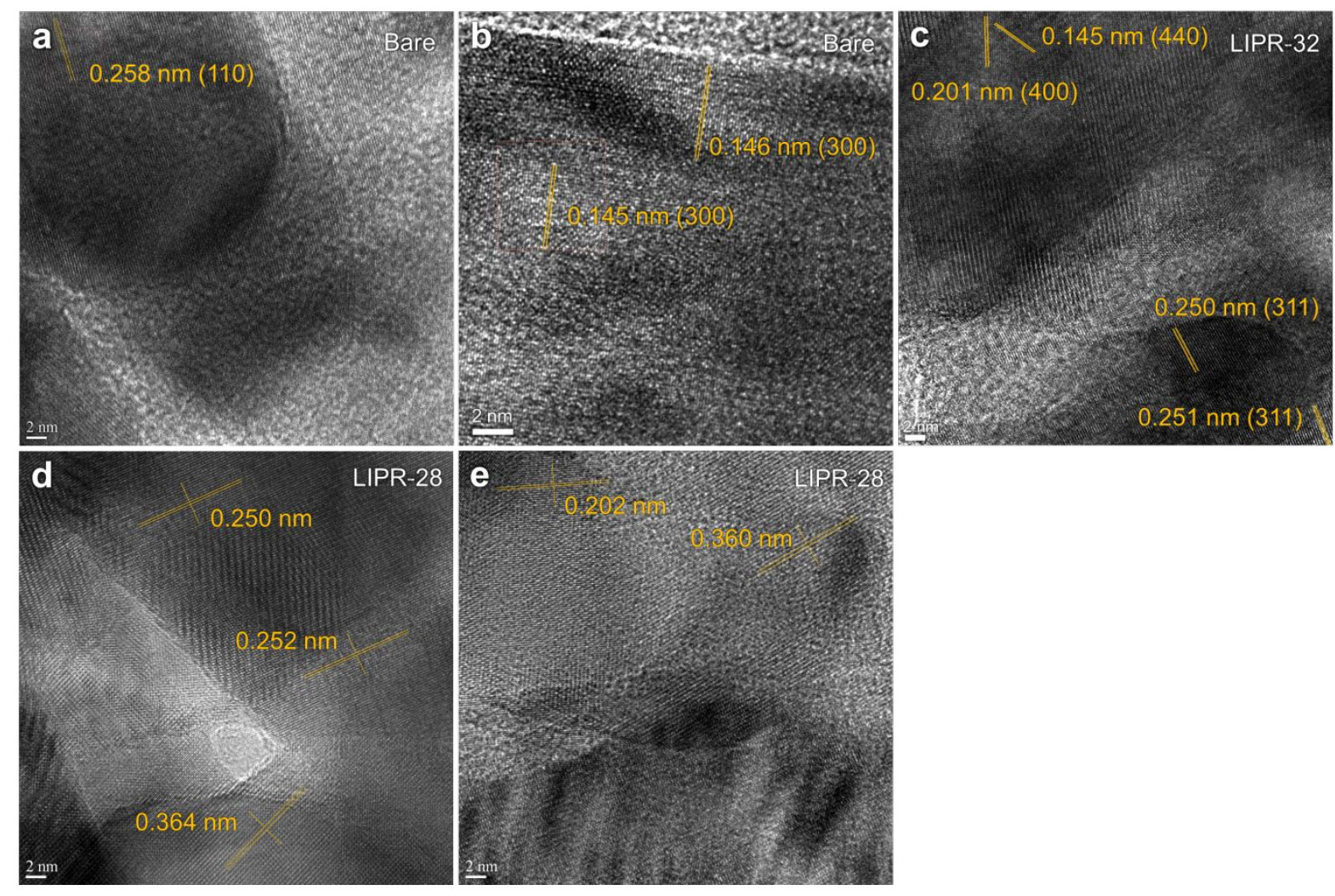

Figure S6. The HR-TEM images of (a)-(b) the bare hematite NRs, (c) the LIPR-32, and (d)-(e) the LIPR-28. 


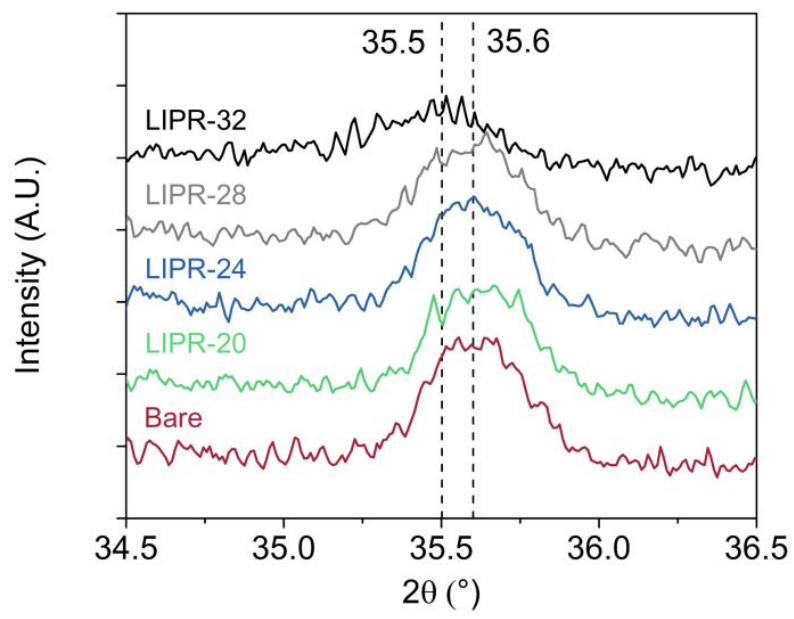

Figure S7. The magnified XRD patterns around $35.5^{\circ}$ of the bare hematite NRs and the LIPR samples. The bare hematite NRs, LIPR-20, LIPR-24, and LIPR-28 exhibited peaks at $35.6^{\circ}$ corresponding to the (110) diffraction direction of hematite, while the LIPR-32 exhibited a peak at $35.5^{\circ}$ corresponding to the (311) direction of magnetite. 


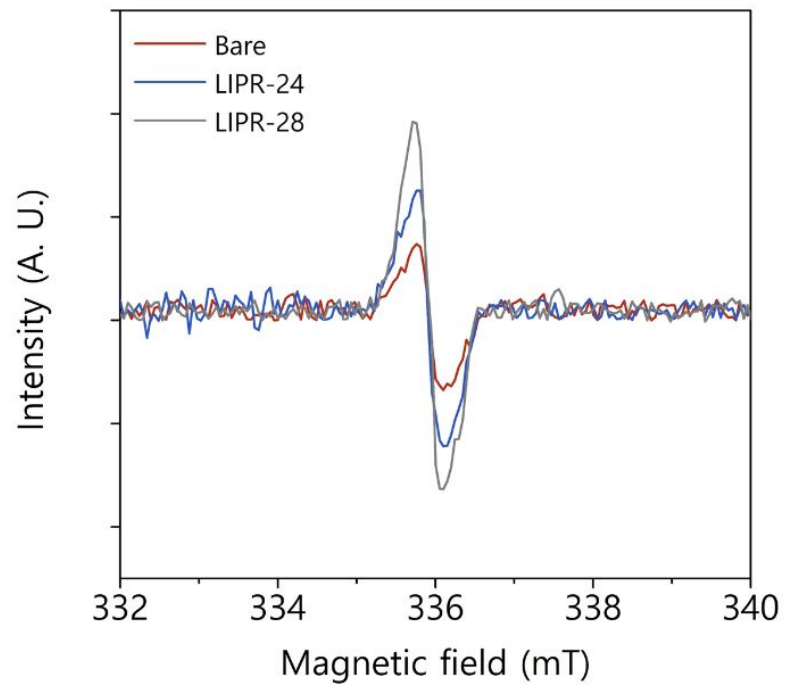

Figure S8. The room temperature ESR spectra of the bare hematite NRs, the LIPR-24, and the LIPR-28. 


\section{Calculation of g-factor}

The g-factor $(g)$ was calculated by the following equation:

$g=h v / \mu_{B} B$

where $h$ is the Planck constant $\left(6.626 \times 10^{-34} \mathrm{~J} \mathrm{~s}\right), v$ is the frequency $\left(9.414 \times 10^{9} \mathrm{~Hz}\right.$ for our condition), $\mu_{B}$ is the Bohr magneton $\left(9.274 \times 10^{-24} \mathrm{~J} \mathrm{~T}^{-1}\right)$, and $B$ is magnetic field. All the samples exhibited the ESR signals at $335.9 \mathrm{mT}$. The value of $g$ at $335.9 \mathrm{mT}$ was calculated to be 2.003 , which suggests evidently the electrons trapped by oxygen vacancies. 